\title{
RELAÇÃO ENTRE STRESS PARENTAL E AS CARATERÍSTICAS SOCIOECONÓMICAS DE PAIS DE CRIANÇAS COM NECESSIDADES ESPECIAIS
}

\author{
Maria Augusta Romão Veiga-Branco \\ Instituto Politécnico de Bragança \\ Reserach in Education and Community Intervention \\ aubra@ipb.pt \\ Maria Isabel Barreiro Ribeiro \\ Instituto Politécnico de Bragança \\ Centro de Investigação de Montanha \\ xilote@ipb.pt \\ Celeste da Cruz Meirinho Antão \\ Instituto Politécnico de Bragança \\ celeste@ipb.pt \\ Zélia Caçador Anastácio \\ Universidade do Minho \\ zeliaf@ie.uminho.pt
}

Fecha de Recepción: 19 Febrero 2018

Fecha de Admisión: 10 Abril 2018

\section{RESUMO}

\section{Introdução}

Existe relação entre stress parental, bem-estar familiar e desenvolvimento bio-psicológico dos filhos. Um risco acrescido de desenvolver patologia físico-psíquica está associado aos progenitores de crianças com necessidades especiais (CNE)

Objetivos: Determinar o nível de stress em pais de CNE e identificar caraterísticas socioeconómicas que possam estar associadas a elevados níveis de stress.

Métodos: Foi realizado um estudo observacional, transversal e analítico, com base numa amostra não aleatória, constituída por 274 pais de CNE em Portugal. Os dados foram recolhidos de abril a outubro de 2017. Como instrumento para a recolha de dados foi utilizado um questionário que incluiu questões de natureza socioeconómica e a Escala de Stress Parental (ESP) constituída por 18 itens, avaliados numa escala de Likert que varia de 1 (discordo totalmente) a 5 (concordo totalmente). 0 score de stress resultou do somatório de todos os itens e foi, posteriormente, classificado tendo em conta os seguintes pontos de corte: Baixo:18-40; moderado: 41-66 e, elevado: 67 a 90.

Resultados: Amostra maioritariamente do género feminino (65\%), com idade média de 40,5 anos $(\mathrm{DP} \pm 8,25)$, dos distritos de Bragança ou Porto, $(73,4 \%)$, com 12 anos de escolaridade 
(56,2\%), casado(a) $(61,3 \%)$, ocupação profissional a tempo inteiro $(71,9 \%)$, rendimento mensal $\leq 1200$ euros/mês (46,7\%). 0 nível de stress parental variou de baixo (10,6\%), moderado (46,7\%) a elevado (42\%). Verificaram-se associações, estatisticamente significativas, entre o nível de stress parental e as variáveis: distrito de residência, estado civil e rendimento do agregado familiar. A proporção de pais com níveis de stress elevados, foi maior em: distritos da Guarda, Viseu, Castelo Branco e Faro; casais em união de facto ou divorciados; em agregados com rendimento mensal $\leq 1$ salário mínimo nacional (557 euros/mês).

Considerações finais: 0 stress parental deve ser foco de implementação de ações promotoras do desenvolvimento saudável das CNE.

Palavras-Chave: stress parental; crianças com necessidades especais; saúde mental

\section{ABSTRACT}

Relationship between Parental Stress and the socioeconomic characteristics of parents of children with special needs.

Introduction: There is a relationship between parental stress, family well-being and bio-psychological development of the children. An increased risk of developing physical-psychological pathology is associated with parents of children with special needs (CSN).

Objetives: To determine the level of stress in CSN parents and to identify socioeconomic characteristics that may be associated with high levels of stress.

Methods: An observational, cross-sectional and analytical study was performed based on a nonrandom sample of 274 CSN parents in Portugal. Data were collected from April to October 2017. As a tool for data collection, a questionnaire was used that included questions of socioeconomic nature and the Parental Stress Scale (PSS) consisting of 18 items, evaluated on a Likert scale that varies from 1 (totally disagree) to 5 (totally agree). The stress score resulted from the sum of all items and, later, it was classified taking into account the following cutoff points: Low: 18-40; moderate: 41-66 and high: 67-90.

Results: Parents that respond to the questionnaire were, mainly, female $(65 \%)$, with a mean age of 40.5 years old $(S D=8.25)$, from Bragança or Porto districts $(73.4 \%)$, with 12 years of schooling $(56.2 \%)$, married $(61.3 \%)$, full-time occupation $(71.9 \%)$, monthly income $\leq 1200$ euros per month $(46.7 \%)$. The level of parental stress ranged from low $(10.6 \%)$, moderate $(46.7 \%)$ to high $(42 \%)$. There were, statistically, significant associations between the level of parental stress and the variables: district of residence, marital status and household income. The proportion of parents with high stress levels was higher in: Guarda, Viseu, Castelo Branco and Faro districts; couples in a stable relation or divorced; in households with monthly income $\leq$ to national minimum wage (557 euros per month). Conclusion: Parental stress should be the focus of implementation of actions promoting the healthy development of CSN.

Keywords: parental stress; children with special needs; mental health

\section{INTRODUÇÃO}

Evidências empíricas destacam o stress psicológico e um nível negativo de bem-estar subjetivo de pais de crianças com necessidades especiais (CNE) (Lim \& Chong, 2017). A literatura sobre pais de CNE revelam altos níveis de stress parental, grande necessidade de cuidados especiais de saúde para as crianças, risco elevado de perturbações psicológicas, sociais e stress financeiro (Chiu, 2013; Jackson, Liang, Frydenberg, Higgins \& Murphy, 2016; Smith \& Grzywacz, 2014). Os pais de CNE enfrentam desafios específicos na realização dos seus papéis de cuidadores, enfrentando riscos acrescidos de desenvolver distúrbios de saúde físicos e ou psíquicos (Gravdal Kvarme, AlbertiniFrüh, Brekke, Gardsjord, Halvorsrud \& Liden, 2016; Hauge, Nes, Kornstad, Kristensen, Irgens, 
Landolt, Eskedal \& Vollrath, 2015; Petcharat \& Liehr, 2017; Ribeiro, Porto \& Vandenberghe, 2013; Redquest, Reinders, Bryden, Schneider \& Fletcher, 2015). Além destas perturbações, os problemas comportamentais das crianças, também contribuem para maior incidência de sintomas de depressão e sentimentos de menor eficácia parental, bem como para 0 aumento dos sintomas depressivos ao longo do tempo (Woodman \& Hauser-Cram, 2013).

Quando os pais experimentam elevados níveis de stress comprometem não só o seu próprio bem-estar, como o da família, e sobretudo o desenvolvimento físico e intelectual dos seus filhos. Um estudo desenvolvido por Caicedo (2015) que teve como objetivos analisar a saúde física (funcionamento físico), saúde mental (funcionamento emocional, social e cognitivo, comunicação e preocupação), funcionamento familiar (atividades quotidianas, relações familiares) e sobrecarga de cuidados, 0 autor concluiu que os pais se sentem fisicamente cansados para fazer o que gostam, e com pouca energia para tarefas domésticas ou atividades sociais. Frequentemente, sentem-se frustrados, ansiosos, zangados, impotentes e sem esperança, têm problemas cognitivos, vivem preocupados com o presente e o futuro da criança e sentem-se socialmente isolados. Partindo destas evidências, o presente trabalho de investigação teve como objetivos determinar o nível de stress em pais de CNE e identificar caraterísticas socioeconómicas que possam estar associadas a elevados níveis de stress.

\section{MÉTODO}

Esta investigação, de carácter transversal, observacional e analítico, teve como população-alvo, pais de CNE em Portugal. A amostra, não probabilística, é constituída por 274 pais e foi recolhida durante no período de abril a outubro de 2017.

Como instrumento para a recolha de dados foi utilizado um questionário que incluiu questões de natureza socioeconómica, nomeadamente, género, idade, local de residência permanente, estado civil, situação profissional, horas de trabalho/semana e rendimento mensal do agregado familiar. Foi, ainda, utilizada a Escala de Stress Parental (ESP), validada para a população portuguesa por Mixão, Leal e Maroco (2010), desenvolvida a partir da escala de Berry e Jones (1995). Esta escala é constituída por 18 itens, avaliados numa escala de Likert que varia de 1 (discordo totalmente) a 5 (concordo totalmente) e descreve sentimentos e perceções sobre ser pai e mãe de CNE. Para calcular 0 score de stress parental, os itens 1, 2, 5, 6, 7, 8, 17 e 18 foram invertidos. 0 score de stress resultou do somatório de todos os itens, e foi, posteriormente, classificado tendo em conta os seguintes pontos de corte: Baixo:18-40; moderado: 41-66 e elevado: 67 a 90.

Após a recolha de dados foi construída uma base no programa IBM Software SPSS versão 24 , na qual foram editados os dados. Estes foram, posteriormente, tratados recorrendo a técnicas estatísticas como sejam: (1) a análise descritiva univariada, nomeadamente, o cálculo de frequências relativas e absolutas nas variáveis de natureza qualitativa e 0 cálculo de medidas de tendência central e de dispersão nas variáveis de natureza quantitativa, e (2) análise bivariada, designadamente, 0 cálculo do teste do qui-quadrado ${ }^{2}$ ) ou em alternativa o teste do qui-quadrado de Monte Carlo () para relacionar as variáveis socioeconómicas com o nível de stress. 0 nível de significância utilizado foi de $5 \%$.

\section{RESULTADOS}

Da totalidade dos inquiridos, a maioria era do género feminino (65\%), possuía em média 40,5 anos de idade (DP $\pm 8,25)$, tinha até 12 anos de escolaridade (56,2\%), era casado(a) (61,3\%), tinha uma ocupação profissional a tempo inteiro $(71,9 \%)$ e residia no distrito de Bragança ou Porto $(73,4 \%)$ (Figura 1). Uma parte significativa, dos agregados familiares, usufruía de um rendimento mensal inferior a 1200 euros/mês (46,7\%) (Tabela 1). 
RELAÇÃO ENTRE STRESS PARENTAL E AS CARATERÍSTICAS SOCIOECONÓMICAS DE PAIS DE CRIANÇAS COM NECESSIDADES ESPECIAIS

Tabela 1 - Caraterização socioeconómica dos pais de CNE

\begin{tabular}{|c|c|c|c|}
\hline \multirow[t]{2}{*}{ Variáveis } & \multirow[t]{2}{*}{ Grupos } & \multicolumn{2}{|c|}{ Frequências } \\
\hline & & Absolutas (n) & Relativas (\%) \\
\hline Género & $\begin{array}{l}\text { Feminino } \\
\text { Masculino }\end{array}$ & $\begin{array}{c}178 \\
96\end{array}$ & $\begin{array}{l}65 \\
35\end{array}$ \\
\hline Classes etárias & $\begin{array}{l}22-40 \text { anos } \\
>40 \text { anos }\end{array}$ & $\begin{array}{l}148 \\
126 \\
\end{array}$ & $\begin{array}{l}54 \\
46 \\
\end{array}$ \\
\hline Estado civil & $\begin{array}{c}\text { Solteiro(a) } \\
\text { Casado(a) } \\
\text { Vive com o parceiro(a) } \\
\text { Divorciado(a) } \\
\text { Viúvo(a) } \\
\end{array}$ & $\begin{array}{c}15 \\
168 \\
67 \\
19 \\
5 \\
\end{array}$ & $\begin{array}{c}5,5 \\
61,3 \\
24,5 \\
6,9 \\
1,8 \\
\end{array}$ \\
\hline $\begin{array}{c}\text { Nível de } \\
\text { escolaridade }\end{array}$ & $\begin{array}{c}\text { Não sabe ler nem escrever } \\
1^{\circ} \text { ciclo } \\
2^{\circ} \text { ciclo } \\
3^{\circ} \text { ciclo } \\
\text { Ensino Secundário } \\
\text { Ensino profissional } \\
\text { Ensino secundário e profissional } \\
\text { Ensino superior }\end{array}$ & $\begin{array}{c}5 \\
15 \\
30 \\
50 \\
54 \\
26 \\
10 \\
84\end{array}$ & $\begin{array}{c}1,8 \\
5,5 \\
10,9 \\
18,2 \\
19,7 \\
9,5 \\
3,6 \\
30,7\end{array}$ \\
\hline $\begin{array}{c}\text { Situação } \\
\text { profissional }\end{array}$ & $\begin{array}{l}\text { Emprego a tempo inteiro } \\
\text { Emprego a tempo parcial } \\
\text { Desempregado(a) } \\
\text { Doméstico(a) } \\
\text { Reformado(a) }\end{array}$ & $\begin{array}{c}197 \\
30 \\
22 \\
22 \\
3 \\
\end{array}$ & $\begin{array}{c}71,9 \\
10,9 \\
8 \\
8 \\
1,1 \\
\end{array}$ \\
\hline Horas Trabalho & $\begin{array}{c}\text { Sem emprego permanente } \\
<40 \text { horas/semana } \\
\geq 40 \text { horas/semana }\end{array}$ & $\begin{array}{c}50 \\
127 \\
97 \\
\end{array}$ & $\begin{array}{l}18,2 \\
46,4 \\
35,4\end{array}$ \\
\hline $\begin{array}{l}\text { Rendimento } \\
\text { agregado familiar }\end{array}$ & $\begin{aligned} & <1 \mathrm{SMN} \\
= & 1 \mathrm{e}<2 \mathrm{SMN} \\
= & 2 \mathrm{e}<3 \mathrm{SMN} \\
= & 3 \mathrm{e}<4 \mathrm{SMN} \\
= & 4 \mathrm{e}<5 \mathrm{SMN} \\
= & 5 \mathrm{e}<6 \mathrm{SMN} \\
& \geq 6 \mathrm{SMN}\end{aligned}$ & $\begin{array}{c}42 \\
86 \\
81 \\
49 \\
7 \\
4 \\
5\end{array}$ & $\begin{array}{c}15,3 \\
31,4 \\
29,6 \\
17,9 \\
2,6 \\
1,5 \\
1,8\end{array}$ \\
\hline Idade (anos) & $\mathrm{Med}$ & ntral & \\
\hline
\end{tabular}

Legenda: SMN - Salário Mínimo Nacional (557€) 
Figura 1 - Distribuição dos pais de CNE por distrito de residência

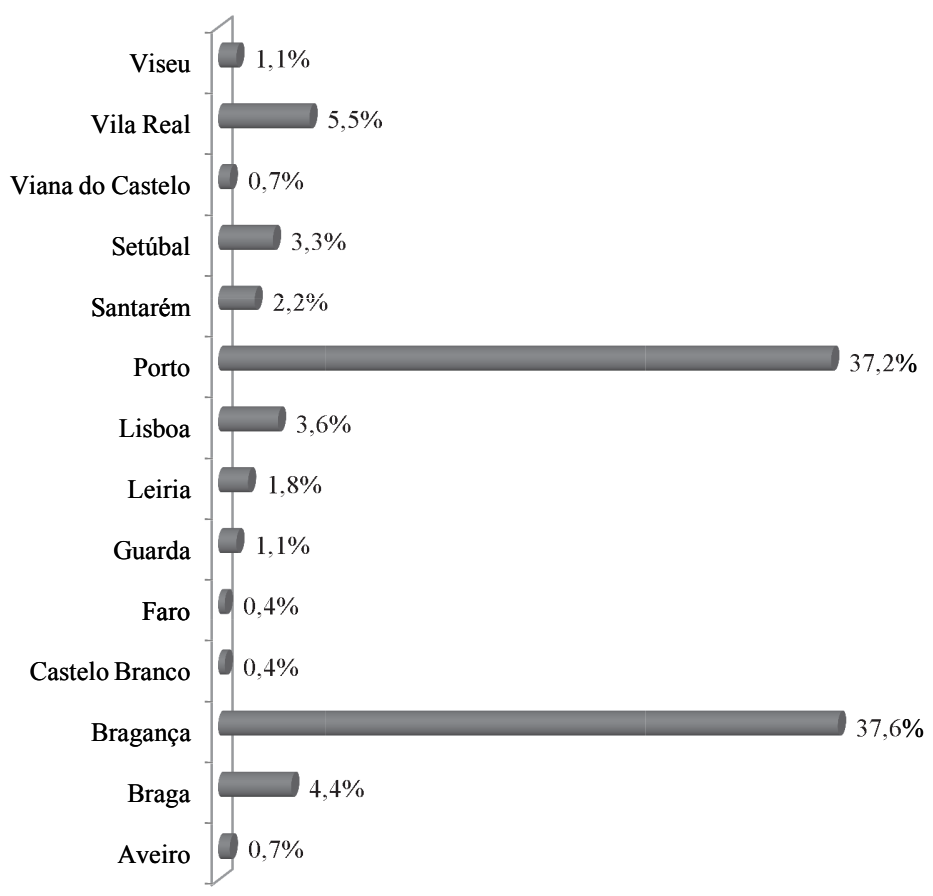

Os distúrbios, doenças ou problemas das crianças incluíam dificuldades de aprendizagem $(35,9 \%)$, perturbações emocionais $(27,5 \%)$ e comportamentais $(25,6 \%)$, deficiência cognitiva ou incapacidade intelectual (35,5\%) transtorno do deficit de atenção e hiperatividade (18,7\%), Autismo $(11,5 \%)$ entre outros (Figura 2$)$ 
Figura 2 - Patologias, problemas ou distúrbios das crianças

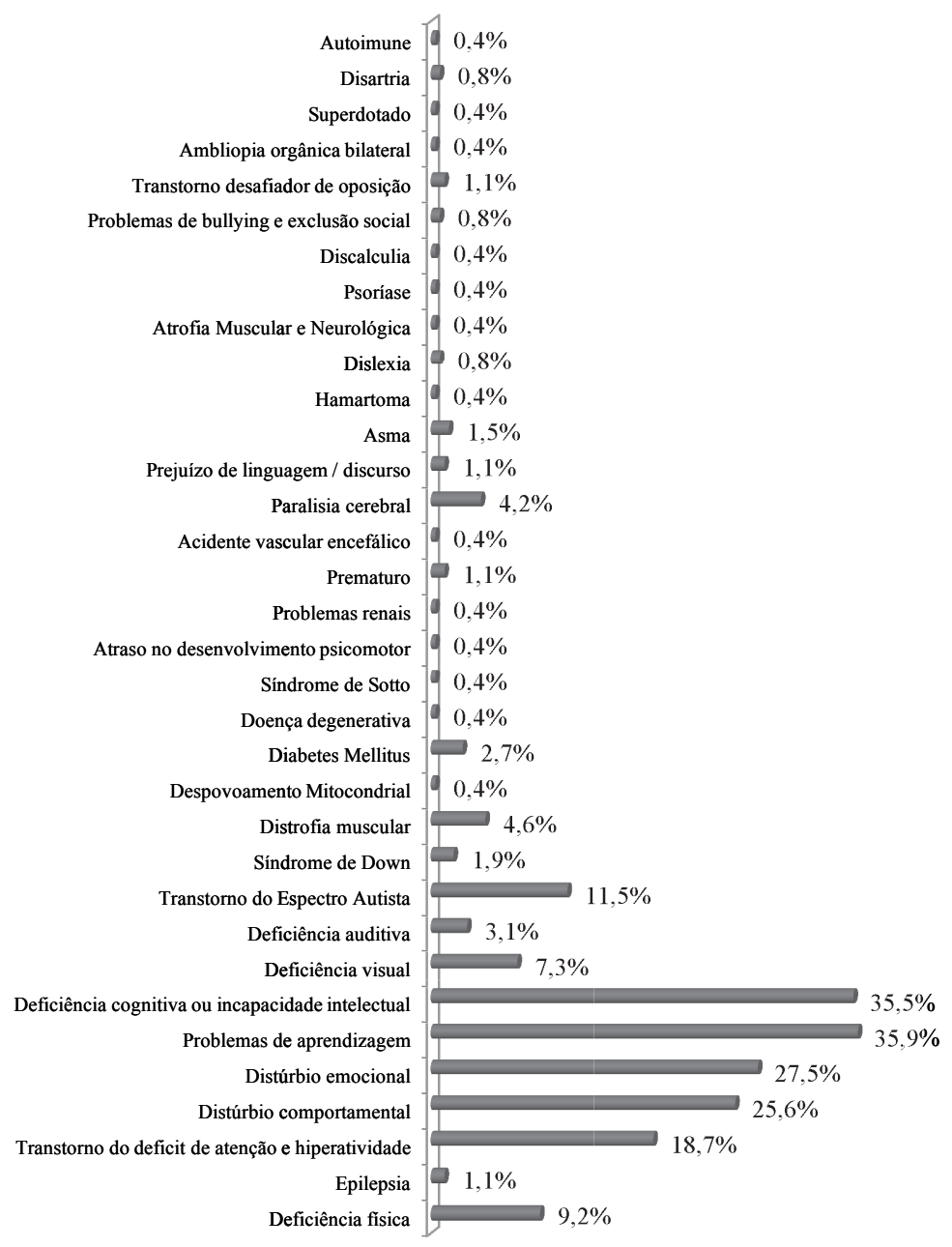
3).

0 nível de stress parental variou de baixo (10,6\%), moderado (46,7\%) a elevado (42\%) (Figura

Figura 3 - Nível de stress dos pais de CNE

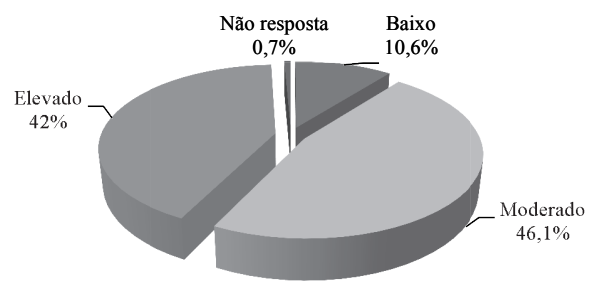


Foram encontradas associações, estatisticamente significativas, entre o nível de stress parental e as variáveis, rendimento do agregado familiar (), distrito de residência ) e estado civil ). A proporção de pais com níveis de stress elevados, foi maior, nos distritos da Guarda, Viseu, Castelo Branco e Faro, nos que viviam em união de facto ou divorciados e entre aqueles que tinham um rendimento mensal inferir a 557 euros/mês.

Os resultados mostraram não existir relação entre o nível de stress dos pais de CNE e as variáveis, género ), idade ), nível de escolaridade ), situação profissional () e horas de trabalho semanais ) (Tabela 2).

Tabela 2 - Relação entre as variáveis socioeconómicas e o nível de stress parental

\begin{tabular}{|c|c|c|c|c|c|}
\hline \multirow[t]{2}{*}{ Variáveis } & \multirow[t]{2}{*}{ Grupos } & \multicolumn{3}{|c|}{ Nível de stress parental (\%) } & \multirow[t]{2}{*}{$p$} \\
\hline & & Baixo & Moderado & Elevado & \\
\hline Género & $\begin{array}{l}\text { Feminino } \\
\text { Masculino }\end{array}$ & $\begin{array}{c}12,4 \\
7,4 \\
\end{array}$ & $\begin{array}{l}49,4 \\
42,6\end{array}$ & $\begin{array}{c}38,2 \\
50 \\
\end{array}$ & 0,140 \\
\hline Classes etárias & $\begin{array}{c}22-40 \text { anos } \\
>40 \text { anos }\end{array}$ & $\begin{array}{c}11,6 \\
9,6 \\
\end{array}$ & $\begin{array}{l}42,2 \\
52,8\end{array}$ & $\begin{array}{l}46,3 \\
37,6 \\
\end{array}$ & 0,224 \\
\hline Estado civil & $\begin{array}{c}\text { Solteiro(a) } \\
\text { Casado(a) } \\
\text { Vive com o parceiro(a) } \\
\text { Divorciado(a) } \\
\text { Viúvo(a) } \\
\end{array}$ & $\begin{array}{c}0 \\
14,9 \\
3 \\
5,6 \\
20 \\
\end{array}$ & $\begin{array}{c}33,3 \\
56,6 \\
33,3 \\
16,7 \\
60 \\
\end{array}$ & $\begin{array}{c}66,7 \\
28,6 \\
63,6 \\
77,8 \\
20 \\
\end{array}$ & $0,000^{(1)(2)}$ \\
\hline $\begin{array}{c}\text { Nível de } \\
\text { escolaridade }\end{array}$ & $\begin{array}{c}\text { Não sabe ler nem escrever } \\
1^{\circ} \text { ciclo } \\
2^{\circ} \text { ciclo } \\
3^{\circ} \text { ciclo } \\
\text { Ensino Secundário } \\
\text { Ensino profissional } \\
\text { Ensino secundário e } \\
\text { profissional } \\
\text { Ensino superior }\end{array}$ & $\begin{array}{c}20 \\
13,3 \\
0 \\
6 \\
22,6 \\
11,5 \\
10 \\
8,4 \\
\end{array}$ & $\begin{array}{c}40 \\
33,3 \\
46,7 \\
44 \\
39,6 \\
46,2 \\
60 \\
55,4 \\
\end{array}$ & $\begin{array}{c}40 \\
53,3 \\
53,3 \\
50 \\
37,7 \\
42,3 \\
\\
30 \\
36,1 \\
\end{array}$ & $0,184^{(2)}$ \\
\hline $\begin{array}{c}\text { Situação } \\
\text { profissional }\end{array}$ & $\begin{array}{c}\text { Emprego a tempo inteiro } \\
\text { Emprego a tempo parcial } \\
\text { Desempregado(a) } \\
\text { Doméstico(a) } \\
\text { Reformado(a) }\end{array}$ & $\begin{array}{c}12,8 \\
0 \\
9,1 \\
4,5 \\
33,3 \\
\end{array}$ & $\begin{array}{c}47,7 \\
43,3 \\
50 \\
40,9 \\
66,7 \\
\end{array}$ & $\begin{array}{c}39,5 \\
56,7 \\
40,9 \\
54,5 \\
0 \\
\end{array}$ & $0,209^{(2)}$ \\
\hline $\begin{array}{c}\text { Horas } \\
\text { Trabalho }\end{array}$ & $\begin{array}{c}\text { Sem emprego permanente } \\
<40 \text { horas/semana } \\
\geq 40 \text { horas/semana }\end{array}$ & $\begin{array}{c}6 \\
11,9 \\
11,5\end{array}$ & $\begin{array}{c}48 \\
43,7 \\
51\end{array}$ & $\begin{array}{c}46 \\
44,4 \\
37,5\end{array}$ & $0,618^{(2)}$ \\
\hline $\begin{array}{l}\text { Rendimento } \\
\text { agregado } \\
\text { familiar }\end{array}$ & $\begin{aligned} & <1 \mathrm{SMN} \\
= & 1 \mathrm{e}<2 \mathrm{SMN} \\
= & 2 \mathrm{e}<3 \mathrm{SMN} \\
= & 3 \mathrm{e}<4 \mathrm{SMN} \\
& \geq 4 \mathrm{SMN}\end{aligned}$ & $\begin{array}{c}4,8 \\
4,7 \\
16,3 \\
18,4 \\
6,3 \\
\end{array}$ & $\begin{array}{l}35,7 \\
52,9 \\
38,8 \\
46,9 \\
87,5 \\
\end{array}$ & $\begin{array}{c}59,5 \\
42,4 \\
45 \\
34,7 \\
6,3 \\
\end{array}$ & $0,001^{(1)}$ \\
\hline $\begin{array}{l}\text { Distrito de } \\
\text { residência }\end{array}$ & $\begin{array}{c}\text { Aveiro } \\
\text { Braga } \\
\text { Bragança } \\
\text { Castelo Branco } \\
\text { Faro } \\
\text { Guarda } \\
\text { Leiria } \\
\text { Lisboa } \\
\text { Porto }\end{array}$ & $\begin{array}{c}50 \\
41,7 \\
2 \\
0 \\
0 \\
0 \\
0 \\
0 \\
18,6\end{array}$ & $\begin{array}{c}0 \\
33,3 \\
57,8 \\
0 \\
0 \\
0 \\
20 \\
40 \\
45,1\end{array}$ & $\begin{array}{c}50 \\
25 \\
40,2 \\
100 \\
100 \\
100 \\
80 \\
60 \\
36,3\end{array}$ & $0,002^{(1)(2)}$ \\
\hline
\end{tabular}




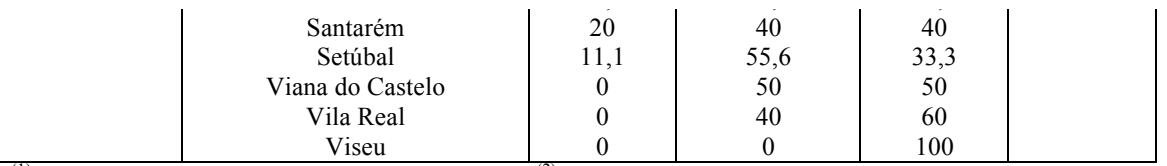

${ }^{(1)}$ Variáveis associadas ao nível de stress parental; ${ }^{(2)}$ Qui-quadrado de Monte Carlo

\section{DISCUSSÃO}

Neste estudo, foram analisadas as relações entre o stress parental e as variáveis sociodemográficas dos pais de CNE. As patologias, distúrbios ou problemas das crianças eram, essencialmente, comportamentais, emocionais e de aprendizagem.

Os resultados desta investigação corroboram a hipótese de que existe relação estatisticamente significativa entre 0 stress parental e o nível de rendimento do agregado familiar. As despesas de saúde e os apoios que os pais procuram para os seus filhos pesam consideravelmente no orçamento da família, facto que é sentido fundamentalmente pelas famílias com rendimentos mais baixos. Estas famílias, na maioria das vezes, não têm capacidade financeira para usufruir dos serviços e ou dos apoios que responderiam em pleno às necessidades dos seus filhos, o que por si só é uma fonte de incapacidade, frustração, impotência e stress.

Os resultados demonstraram que o local de residência está associado ao stress parental. A disponibilidade e 0 acesso a serviços de apoio social, mobilidade e ou de saúde têm sido dificuldades sentidas pelas famílias que vivem em zonas do interior do país, mais despovoadas e carenciadas, nomeadamente, Viseu e Guarda, dificuldades que os pais de CNE sentem com mais intensidade. A perceção de que os seus filhos não são devidamente acompanhados gera sentimentos de preocupação e de impotência, sempre presentes no dia-a-dia destes pais.

Por fim, verificou-se que 0 estado civil dos pais está relacionado com o stress parental e que eram os pais que viviam em regime de coabitação ou divorciados os que experimentavam níveis de stress mais elevados. Resultados semelhantes foram obtidos por Brobst, Clopton e Hendrick (2009). Segundo estes autores os pais de CNE vivenciam níveis de stress mais levados o que aumenta a probabilidade de divórcio e/ou rutura da relação entre o casal.

No presente estudo verificou-se que o nível de stress parental era independente do género, do nível de escolaridade e da situação profissional. Contudo, a literatura evidencia que os níveis de stress, ansiedade e depressão das mães são mais elevados do que os dos pais (Moes, Koegel, Schreibman, \& Loos, 1992; Weis, 2002). Já, Kabasakal, Girli, Totan e Aysan (2012) concluíram que os pais com menor nível de escolaridade vivenciavam mais sentimentos negativos e de stress, em comparação com os pais com nível de escolaridade mais elevado. Por fim, McConkey, Truesdale Kennedy, Chang, Jarrah e Shukri (2008) e Brobst, Clopton e Hendrick (2009) alegam que a probabilidade de interrupção da carreira e de sofrer de stress agudo, é maior em pais de CNE comparativamente aos pais de filhos que não necessitam de cuidados especiais.

Neste trabalho de investigação verificou-se que o estado de saúde, física e mental, dos filhos acarreta uma sobrecarga emocional, social e económica para os pais. Resultados semelhantes foram obtidos por Caiedo (2015), Chiu (2013), Jackson et al. (2016) e Smith \& Grzywacz (2014), Neste sentido, Ki e Joanne (2014), Wakimizu e Fujioka (2016) defendem que é fundamental oferecer, planear e proceder ao aconselhamento, apoio e recursos às famílias e às CNE por forma a aprenderem a lidar mais eficazmente com as suas limitações e ou dificuldades. Tal como Smith e Grzywacz (2014) argumentam, existem stressores adicionais, intrínsecos à paternidade de uma CNE que podem deteriorar a saúde, física e mental dos pais, a longo prazo, não instituindo, contudo, uma barreira à mudança. 


\section{CONCLUSÕES}

Partindo dos objetivos propostos, foi determinado que o nível de stress em pais de CNE, se apresenta moderado e elevado, e, foram identificados: o nível de rendimento do agregado familiar, o local de residência e o estado civil, como caraterísticas socioeconómicas associadas a elevados níveis de stress.

0 stress parental deve ser um indicador de saúde pública a ter em conta, na implementação de ações que promovam não só o bem-estar subjetivo destes pais, como também e sobretudo, 0 desenvolvimento saudável das CNE. A identificação e sinalização das famílias com crianças que necessitam de atenção e cuidados especiais, junto das instituições de educação e saúde competentes, facilita 0 acesso a intervenções multidisciplinares que atendam às necessidades das crianças e das famílias mais vulneráveis.

\section{AGRADECIMENTOS}

Este trabalho foi desenvolvido no âmbito do Projeto Erasmus+ BUILDING BRIDGES: PROMOTING SOCIAL INCLUSION AND WELLBEING FOR FAMILIES OF CHILDREN WITH SPECIAL NEEDS (PSI-WEL) - KA2 - Cooperation for Innovation and the Exchange of Good Practices Strategic Partnerships for adult education - Form hash code: D54537E3B63E4B91. (IPB Coord: Veiga-Branco, M.A.R.).

\section{REFERÊNCIAS BIBLIOGRÁFICAS}

Berry, J.D., \& Jones, W.H. (1995). The Parental Stress Scale: initial psychometric evidence. Journal of Social and Personal Relationships, 12, 463-472.

Brobst, J. B., Clopton, J. R., \& Hendrick, S. S. (2009). Parenting Children with Autism Spectrum Disorders: The Couple's Relationship. Focus on Autism and Other Developmental Disabilities, 24, 38-49.

Caicedo, C. (2015). Families with special needs children: family health, functioning, and care burden. J Am Psychiatr Nurses Assoc., 20(6), 398-407. doi: 10.1177/1078390314561326.

Chiu, E.C. (2013). Preliminary study: Taiwanese mothers' experiences of children with sensory processing disorder. J Nurs Res. 21(3), 219-23. doi: 10.1097/jnr.0b013e3182a0afd4.

Gravdal Kvarme, L., Albertini-Früh, E., Brekke, I., Gardsjord, R., Halvorsrud, L., \& Liden, H. (2016). On duty all the time: health and quality of life among immigrant parents caring for a child with complex health needs. J Clin Nurs. 25(3-4), 62-71. doi: 10.1111/jocn.13056.

Hauge,. LJ., Nes, R.B., Kornstad, T., Kristensen, P., Irgens, L.M., Landolt, M.A., Eskedal, L.T., \& Vollrath, M.E. (2015). Maternal Sick Leave Due to Psychiatric Disorders Following the Birth of a Child With Special Health Care Needs. J Pediatr Psychol., 40(8), 804-13. doi: 10.1093/jpepsy/jsv034.

Jackson, A.C., Liang, R.P., Frydenberg, E., Higgins, R.O., \& Murphy, B.M. (2016). Parent education programmes for special health care needs children: a systematic review. J Clin Nurs., 25(11-12), 1528-47. doi: 10.1111/jocn.13178.

Kabasakal, Z., Girli, A., Totan, T., \& Aysan, F. (2012). Psychiatric symptoms as predictors of life satisfaction in parents with handicapped children. Journal of Theory and Practice in Education, 8 (2).

Ki, Y.W., \& Joanne, C.C.Y. (2014). Stress and Marital Satisfaction of Parents with Children with Disabilities in Hong Kong. Psychology, 5 (05), 349-357.

Lim. K.K., \& Chong, W.H. (2017). Moderating effect of child's autism spectrum disorder (ASD) diagnosis on benefit finding and negative affect of parents. Am J Orthopsychiatry. 87(3), 357-364. doi: 10.1037/ort0000251. 
McConkey, R., Truesdale-Kennedy, M., Chang, M. Y., Jarrah, S., \& Shukri, R. (2008). The Impact on Mothers of Bringing up a Child with Intellectual Disabilities: A Cross-Cultural Study. International Journal of Nursing Studies, 45, 65-74.

Mixão, M.L., Leal, L., \& Maroco, J. (2010). Escala de stress parental. In I. Leal \& J. Maroco, Eds. Avaliação em sexualidade e parentalidade. Porto: LivPsic.

Moes, D., Koegel, R.L., Schreibman, L., \& Loos, L.M. (1992). Stress profiles for mothers and fathers of children with autism. Psychol Report., 71(3- 2), 1272-1274.

Petcharat, M. \& Liehr, P. (2017). Mindfulness training for parents of children with special needs: Guidance for nurses in mental health practice. J Child Adolesc Psychiatr Nurs., 30(1), 35-46. doi: 10.1111/jcap.12169.

Redquest, B.K., Reinders, N., Bryden, P.J., Schneider, M., \& Fletcher, P.C. (2015). Raising a child with special needs: the perspective of caregivers. Clin Nurse Spec., 29(3): E8-15. doi: 10.1097/NUR.0000000000000122.

Ribeiro, M.F.M., Porto, C.C. \& Vandenberghe, L. (2013). Estresse parental em famílias de crianças com paralisia cerebral: revisão integrativa. Ciência \& Saúde Coletiva, 18 (6), 1705-1715.

Smith, A.M., \& Grzywacz, J.G. (2014). Health and well-being in midlife parents of children with special health needs. Fam Syst Health., 32(3):303-12. doi: 10.1037/fsh0000049.

Wakimizu, R., \& Fujioka, H. (2016). Analysis of the Issues and Needs of Parents of Children With Developmental Disabilities in Japan Using Focus Group Interviews J Nurs Res., 24(1):68-78. doi: $10.1097 /$ jnr.0000000000000093.

Weiss, J.M. (2002). Hardiness and social support as predictors of stress in mothers of typical children, children with autism, and children with mental retardation. Autism, 6(1), 115-130. doi:10.1177/136236130200 6001009

Woodman, A.C., \& Hauser-Cram, P. (2013). The role of coping strategies in predicting change in parenting efficacy and depressive symptoms among mothers of adolescents with developmental disabilities. J Intellect Disabil Res., 57(6):513-30. doi: 10.1111/j.1365-2788.2012.01555.x. 